\title{
EMBEDDINGS OF THE MAXIMAL QUOTIENT RING OF A VON NEUMANN REGULAR RING INTO ITS COMPLETION WITH RESPECT TO A RANK FUNCTION
}

\author{
ANNIE VOGEL \\ (Communicated by Donald Passman)
}

\begin{abstract}
Let $R$ be a commutative von Neumann regular ring. A pseudorank function on $R$ is characterized by a probability measure on the spectrum of $R$. We exhibit two commutative von Neumann regular rings $R$ and $R^{\prime}$ with the same spectrum $S$, and two rank functions $N$ and $N^{\prime}$ on $R$ and $R^{\prime}$, corresponding to the same probability measure on $S$, and such that the maximal right quotient ring $Q_{\max }(R)$ embeds in the $N$-completion $\bar{R}$ of $R$ while $Q_{\max }\left(R^{\prime}\right)$ does not embed in $\overline{R^{\prime}}$.
\end{abstract}

Let $R$ be a von Neumann regular ring, and let $N$ be a rank function on $R$. Let $\bar{R}$ be the $N$-completion of $R ; \bar{R}$ is then a regular right and left self-injective ring and one may ask whether the maximal right quotient ring $Q_{\max }(R)$ of $R$ embeds in $\bar{R}$. Sufficient conditions are already known $[\mathbf{1}, \mathbf{2}]$.

Whenever the ring $R$ is commutative, there exists a one-to-one correspondence between rank functions on $R$ and probability measures on the spectrum of $R$ [3]. So we might expect that the condition " $Q_{\max }(R)$ is isomorphic to a subring of $\bar{R}$ " only depends on the spectrum of $R$ and on the rank function. Actually the answer is negative and we prove:

PROPOSITION 1. There exists a commutative von Neumann regular ring $R^{\prime}, a$ rank function $N$ on $R^{\prime}$ and a regular subring $R$ of $R^{\prime}$ such that

$\operatorname{Spec} R=\operatorname{Spec} R^{\prime}$,

$Q_{\max }(R)$ is isomorphic to a subring of the $N$-completion of $R$, and

$Q_{\max }\left(R^{\prime}\right)$ is not isomorphic to any subring of the $N$-completion of $R^{\prime}$.

Let $K$ be a field, and let $R$ be the set of all stationary sequences of elements of $K$. Then $R$ is a regular subring of $K^{\mathbf{N}}$ and may be viewed as the set of all continuous maps of the one point compactification $\mathbf{N} \cup \infty$ of $\mathbf{N}$ with values in the discrete field $K$.

For any element $x$ of $\mathbf{N} \cup \infty$, let $\mathfrak{p}_{x}$ be the ideal of all sequences vanishing at $x$. The map $x \leadsto \mathfrak{p}_{x}$ realizes a homeomorphism of $N \cup \infty$ onto Spec $R$.

Let $Q$ be the set of all sequences of elements of $K$. It is easy to see that $Q$ is a regular self-injective ring and that $R$ is essential in $Q$. Hence $Q$ is the maximal quotient ring of $R$.

Let $N$ be a rank function on $R$. For any element $a$ of $R$, set

$$
U(a R)=\{\mathfrak{p} \in \operatorname{Spec} R / a R \not \subset \mathfrak{p}\} \text { and } \mu(U(a R))=N(a) .
$$

Received by the editors October 20, 1986 and, in revised form, January 6, 1987.

1980 Mathematics Subject Classification (1985 Revision). Primary 16A30; Secondary 16A08.

Key words and phrases. Von Neumann regular ring, $N$-completion, maximal quotient ring. 
Then $\mu$ extends to a probability measure on $\operatorname{Spec} R$, the support of which is $\operatorname{Spec} R$ [3]; Spec $R$ being countable, $\mu$ is characterized by the measure of points. For all $n$ in $\mathbf{N}$, set

$$
a_{n}=\mu(n)=\mu\left(\mathfrak{p}_{n}\right) \quad \text { and } \quad a_{\infty}=\mu(\infty)=\mu\left(\mathfrak{p}_{\infty}\right)
$$

Then

$$
\sum_{n \in \mathbf{N}} a_{n}+a_{\infty}=1
$$

Remark 1. Since the support of $\mu$ is $\operatorname{Spec} R, a_{n}$ is positive for all $n$.

PROPOSITION 2. Let $N$ be a rank function on $R$ such that $a_{\infty} \neq 0$. Then $Q$ is isomorphic to a subring of the $N$-completion $\bar{R}$ of $R$ if and only if $K$ is finite.

Proof. Suppose $K$ is infinite, and suppose that $Q$ is isomorphic to a subring of $\bar{R}$. Then there exists a rank function $N^{\prime}$ on $Q$ which extends $N$, and such that $R$ is dense in $Q$ with respect to $N^{\prime}$.

Let $x=\left(x_{n}\right)$ be a sequence of distinct elements of $K$. Given any $\varepsilon>0$, there exists an element $y=\left(y_{n}\right)$ of $R$ such that $N^{\prime}(x-y)<\varepsilon$.

Since $\left(y_{n}\right)$ is a stationary sequence, there exists an integer $p$ such that $\forall n>p$ $x_{n}-y_{n} \neq 0$. Set

$$
e_{n}= \begin{cases}1 & \text { if } n>p \\ 0 & \text { otherwise }\end{cases}
$$

The element $e=\left(e_{n}\right)$ is an idempotent in $R$ and the ideal $e Q$ is included in $(x-y) Q$. This yields $N^{\prime}(e)<N^{\prime}(x-y)<\varepsilon$. We have

$$
N^{\prime}(e)=N(e)=1-N(1-e)=1-\left(a_{0}+\cdots+a_{p-1}\right) .
$$

Hence $N^{\prime}(e)>a_{\infty}$, which is a contradiction if $a_{\infty}$ is a nonzero element.

Suppose $K$ is finite and let $\mathcal{U}$ be a nonconvergent ultrafilter in $\mathbf{N}$.

For each element $x=\left(x_{n}\right)$ of $Q$, set

$$
\begin{gathered}
P_{x}=\left\{n \in \mathrm{N} / x_{n} \neq 0\right\}, \\
N^{\prime}(x)= \begin{cases}\mu\left(P_{x}\right)+a_{\infty} & \text { whenever } P_{x} \in \mathcal{U}, \\
\mu\left(P_{x}\right) & \text { otherwise. }\end{cases}
\end{gathered}
$$

Obviously, $N^{\prime}$ is a rank function on $Q$. Moreover, if $x=\left(x_{n}\right)$ is an element of $R$, then

$$
N(x)= \begin{cases}\mu\left(P_{x}\right)+a_{\infty} & \text { whenever } x_{\infty} \neq 0, \\ \mu\left(P_{x}\right) & \text { otherwise, }\end{cases}
$$

and $x_{\infty}=0$ if and only if $P_{x}$ is a bounded subset of $\mathbf{N}$, which means that $P_{x}$ is not an element of $U$. So $N^{\prime}$ extends $N$.

Let $x=\left(x_{n}\right)$ be an element of $Q$. Since $K$ is finite, the family $P_{x}(\alpha)=\{n \in$ $\left.\mathbf{N} / x_{n}=\alpha\right\}_{\alpha \in K}$ is a finite partition of $\mathbf{N}$ and there exists a unique element $\alpha$ in $K$ such that $P_{x}(\alpha)$ is an element of $\mathcal{U}$.

Let $\varepsilon$ be a positive number. The series $\sum_{i=1}^{\infty} a_{i}$ being convergent, there exists an integer $p$ such that $\sum_{i=p}^{\infty} a_{i}<\varepsilon$.

Set

$$
y_{n}= \begin{cases}x_{n} & \text { if } n<p \\ \alpha & \text { otherwise }\end{cases}
$$


Then $y=\left(y_{n}\right)$ is an element of $R$ and $P_{x-y}$, which is disjoint from $P_{x}(\alpha)$, and does not belong to $U$. So $N^{\prime}(x-y)=\mu\left(P_{x-y}\right)$.

On the other hand, $P_{x-y}$ is included in the subset $\{n \in \mathrm{N} / p<n\}$, so that $\mu\left(P_{x-y}\right)<\sum_{i=p}^{\infty} a_{i}$. Thus $R$ is dense in $Q$ with respect to $N^{\prime}$. Then $Q$ is isomorphic to a dense subring of $\bar{R}$.

REMARK 2. Whenever $a_{\infty}$ is nul,

$$
N\left(\mathfrak{p}_{\infty}\right)=\sup \left\{N(x) x \in \mathfrak{p}_{\infty}\right\}=1 \text {. }
$$

So every essential ideal of $R$ has norm one, and with $N$ being countably additive, $Q_{\max }(R)$ is isomorphic to a subring of $\bar{R}$.

PrOOF OF PROPOSITION 1. Let us consider two fields $K$ and $K^{\prime}$ with the following properties: $K$ is finite, $K^{\prime}$ is infinite, and $K$ is included in $K^{\prime}$. Let $R$ (and $R^{\prime}$ ) be the ring of all stationary sequences of elements of $K$ (of $K^{\prime}$ ); $R$ and $R^{\prime}$ are commutative von Neumann regular rings and they have the same spectrum $\mathbf{N} \cup \infty$. A probability measure on $\mathbf{N} \cup \infty$, with support precisely $\mathbf{N} \cup \infty$ and for which $a_{\infty}$ is nonzero, induces two rank functions $N$ and $N^{\prime}$ on $R$ and $R^{\prime}$ which are equal on $R$. Since $a_{\infty}$ is a nonzero element, $Q_{\max }(R)$ is isomorphic to a subring of the $N$-completion $\bar{R}$ of $R$, while $Q_{\max }\left(R^{\prime}\right)$ is not isomorphic to any subring of $\overline{R^{\prime}}$.

REMARK 3. Whenever $K$ is finite, the embedding of $Q_{\max }(R)$ in $\bar{R}$ is not unique: it depends on the choice of a nonconvergent ultrafilter on $\mathbf{N}$.

\section{REFERENCES}

1. K. R. Goodearl and D. Handelman, Simple self injective rings, Comm. Algebra 3 (1975), 797-834.

2. J. M. Goursaud and L. Jeremy, Sur l'enveloppe injective des anneaux réguliers, Comm. Algebra 3 (1975), 763-779.

3. A. Vogel, Mesures de probabilité sur le spectre d'un anneau régulier et applications, C. R. Acad. Sci. Paris 289 (1979).

Institut de Mathématiques et D'Informatique, 2, Rue de la Houssinière, 44072 Nantes Cedex, France 